\title{
Occult follicular lymphoma in a swollen regional lymph node of gastric schwannoma
}

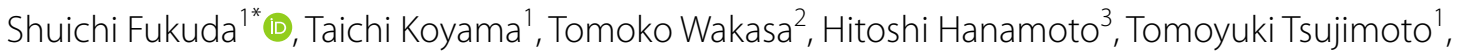
Atsushi Gakuhara ${ }^{1}$, Hideo Tomihara ${ }^{1}$, Katsuya Ohta ${ }^{1}$, Kotaro Kitani ${ }^{1}$, Kazuhiko Hashimoto ${ }^{1}$, Hajime Ishikawa ${ }^{1}$, Jin-ichi Hida', Masao Yukawa', Yoshio Ohta² and Masatoshi Inoue'

\begin{abstract}
Background: Regional lymphadenopathy is more commonly noted in gastric schwannomas than in other gastric submucosal tumors. Most of the swollen lymph nodes associated with gastric schwannomas are non-metastatic lymphadenopathy.

Case presentation: A 69-year-old Japanese woman was referred to our hospital with a chief complaint of abdominal discomfort. Contrast-enhanced computed tomography (CT) of the abdomen revealed an extraluminal tumor with heterogeneous enhancement at the middle stomach on the lesser curve, accompanied with one swollen lymph node approximately $10 \mathrm{~mm}$ in size and several small lymph nodes in the perigastric region. These lymph nodes were flat; therefore, we considered them to be non-metastatic. The main tumor was removed via wedge resection. Soft and slightly swollen lymph nodes, which were compatible with the lymph nodes noted in the preoperative CT, were found near the main tumor in the fatty tissue at the lesser curvature of the stomach. An excisional biopsy of the largest lymph node was performed for the diagnosis. Based on pathological findings, a diagnosis of gastric schwannoma and follicular lymphoma (FL) was confirmed. The patient is doing well without recurrence of either the gastric schwannoma or FL 28 months postsurgery.
\end{abstract}

Conclusions: The present report detailed an extremely rare case of $\mathrm{FL}$ coincidentally discovered in the swollen regional lymph node of gastric schwannoma.

Keywords: Intraoperative pathological diagnosis, Lymphadenopathy, Lymphoid cuff, Malignant lymphoma, Submucosal tumor

\section{Background}

Gastric schwannomas are rare mesenchymal tumors, accounting for approximately $0.2 \%$ of all gastric tumors [1]. Recent studies reported that a computed tomography (CT) finding of gastric schwannoma demonstrated regional lymphadenopathy more frequently than other gastric submucosal tumors (SMTs): gastric schwannoma, $29.0 \%(\geq 20 \mathrm{~mm})$ and $75.0 \%(\geq 50 \mathrm{~mm})$; gastric gastrointestinal stromal tumor, $3.5 \%(\geq 20 \mathrm{~mm})$ and $5.1 \%$

\footnotetext{
*Correspondence: s.f4911@nifty.com

1 Department of Gastroenterological Surgery, Kindai University Nara

Hospital, 1248-1, Otoda-cho, Ikoma, Nara 630-0293, Japan

Full list of author information is available at the end of the article
}

( $\geq 50 \mathrm{~mm}$ ); and gastric leiomyoma, $0.0 \%$ (both $\geq 20 \mathrm{~mm}$ and $\geq 50 \mathrm{~mm}$ ) $[2,3]$. The majority of schwannomas are benign; only a very small proportion is considered malignant with a potential to metastasize [4]. Among the gastric schwannoma patients with swollen lymph nodes, it is extremely rare to actually present with pathological lymph node metastasis [5-7]. Thus, the majority of swollen lymph nodes associated with gastric schwannomas are non-metastatic lymphadenopathy. Here we present an extremely rare case of a patient with a follicular lymphoma (FL) coincidentally discovered in the swollen regional lymph node of gastric schwannoma. 


\section{Case presentation}

A 69-year-old Japanese woman was referred to our hospital with a chief complaint of abdominal discomfort. She had no history of smoking and was a social drinker. She also had a history of hyperlipidemia, depression, and uterine myoma. Body temperature was normal. Physical examination revealed no swelling of superficial lymph nodes. The patient had regular bowel movements with normal stools. She had no B symptoms, including fever, night sweats, or weight loss. Laboratory data were unremarkable: white blood cell (WBC) count, 5890/ $\mu \mathrm{L}$; hemoglobin, $13.2 \mathrm{~g} / \mathrm{dL}$; platelet count, $254,000 / \mu \mathrm{L}$; and lactate dehydrogenase (LDH) level, $217 \mathrm{U} / \mathrm{L}$. Tumor markers, including carcinoembryonic antigen and carbohydrate antigen 19-9, were within normal ranges. The serum soluble interleukin-2 receptor (sIL-2R) level was also within normal ranges.

Abdominal ultrasonography revealed a well-circumscribed hypoechoic mass, contiguous with the proper muscle layer, approximately $70 \mathrm{~mm}$ in size, located at the middle stomach on the lesser curve (Fig. 1a). Contrast-enhanced CT of the abdomen demonstrated an extraluminal tumor with heterogeneous enhancement at the middle stomach on the lesser curve, accompanied with one swollen lymph node approximately $10 \mathrm{~mm}$ in size and several small lymph nodes in the perigastric region (Fig. 1b, c). These lymph nodes were flat; therefore, we considered them to be non-metastatic. No liver metastasis, peritoneal dissemination, pleural fluid, ascites, or splenomegaly was observed. Endoscopy indicated compression of the anterior wall of the gastric body along with normal mucosa (Fig. 1d). Several biopsy specimens were taken, which showed evidence of erosive hyperplastic gastritis. Endoscopic ultrasonography and endoscopic ultrasound-guided fine needle aspiration were not performed for the diagnosis because a SMT greater than $50 \mathrm{~mm}$ in size is an indication for surgery regardless of the preoperative definitive diagnosis [8].

Surgical exploration of the abdomen revealed no evidence of ascites or metastasis to the liver or peritoneum. An extraluminal tumor arising from the middle body of the stomach on the lesser curve was noted. The tumor was removed via wedge resection. Soft and slightly swollen lymph nodes, which were compatible with the lymph
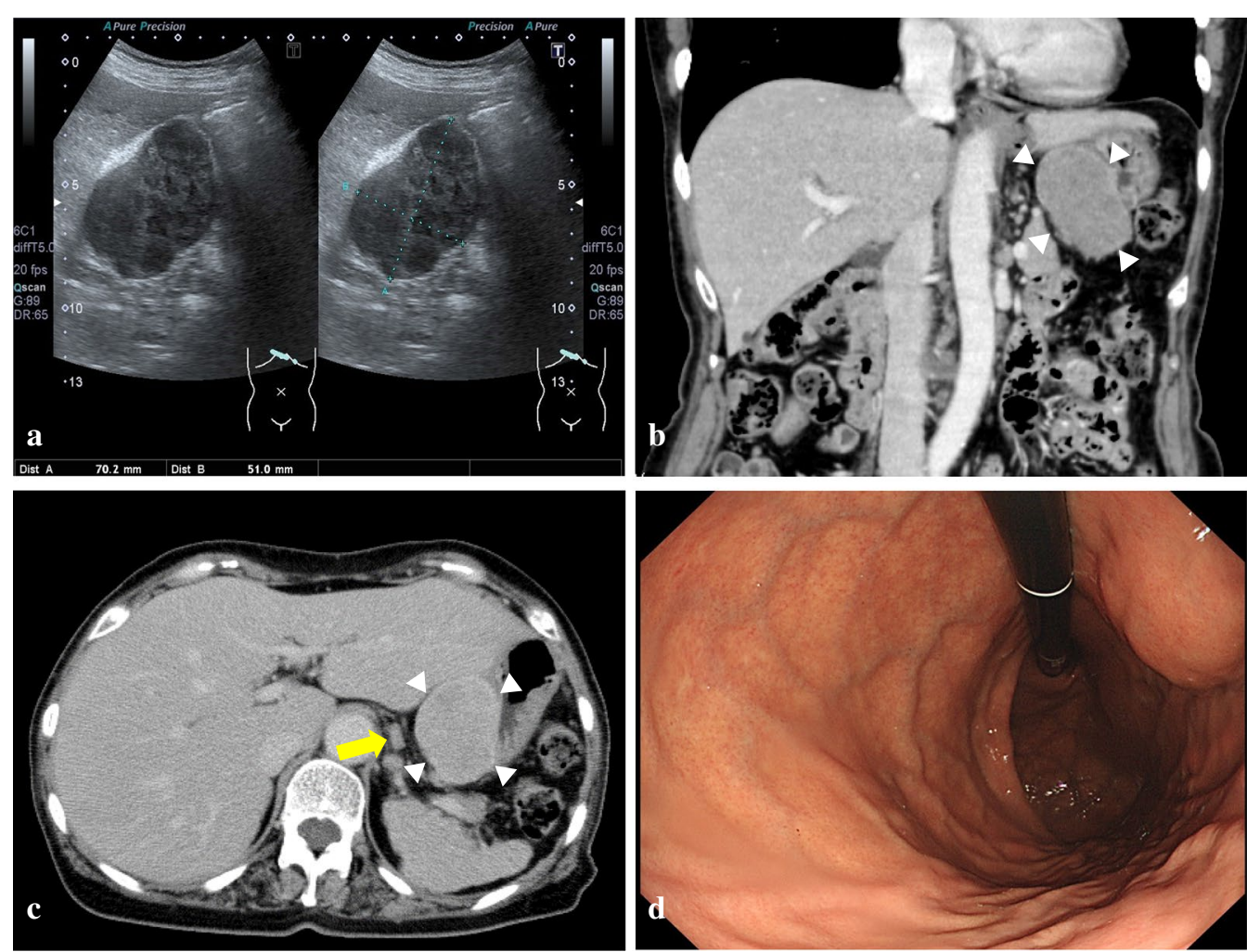

Fig. 1 a Abdominal ultrasonography revealing a well-circumscribed hypoechoic mass, contiguous with the proper muscle layer, approximately $70 \mathrm{~mm}$ in size, located at the middle stomach on the lesser curve. b, c Contrast-enhanced CT of the abdomen showing an extraluminal tumor with heterogeneous enhancement at the middle stomach on the lesser curve (white arrowheads), accompanied with one swollen lymph node approximately $10 \mathrm{~mm}$ in size (yellow arrow) and several small lymph nodes in the perigastric region. $\mathbf{d}$ Endoscopy showing compression of the anterior wall of the gastric body along with normal mucosa 
nodes noted in the preoperative $\mathrm{CT}$, were found near the main tumor in the fatty tissue at the lesser curvature of the stomach. An excisional biopsy of the largest lymph

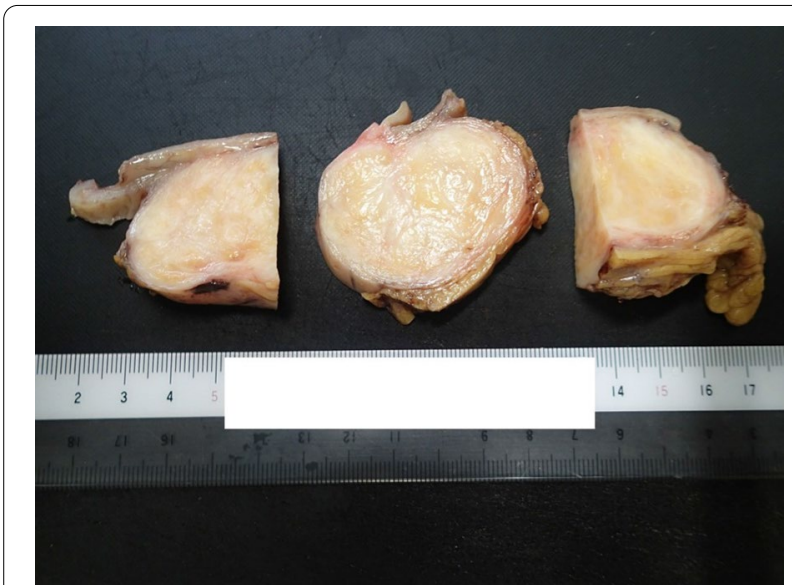

Fig. 2 The cut surface of the tumor revealing a well-circumscribed yellowish-white solid mass node, likely compatible with the swollen lymph node seen in the preoperative $\mathrm{CT}$, was performed for the diagnosis. At this point, we believed these lymph nodes to represent reactive lymphadenopathy.

The resected specimen was $80 \times 60 \times 50 \mathrm{~mm}$ in size. The tumor grew exophytically. The cut surface of the tumor revealed a well-circumscribed yellowish-white solid mass (Fig. 2). Hematoxylin-eosin staining disclosed a bundle-like growth of the spindle-shaped tumor cells with acidophilic cytoplasm (Fig. 3a). These were composed predominantly of tumor cells arranged haphazardly (Antoni type B) and secondarily of tumor cells with nuclear palisading (Antoni type A). A peritumoral lymphoid cuff was recognized (Fig. 3a). The mitotic count was 0 to 1 per 50 high-power fields (HPFs). The tumor contained no necrosis and atypical mitosis was not identified. The resection margins were free of tumor cells. Immunohistochemical staining revealed that the tumor was negative for KIT, CD34, and desmin and positive for $\mathrm{S}-100$ protein (Fig. $3 \mathrm{~b}-\mathrm{e}$ ). The MIB-1 labeling index of the tumor cells was $1 \%$
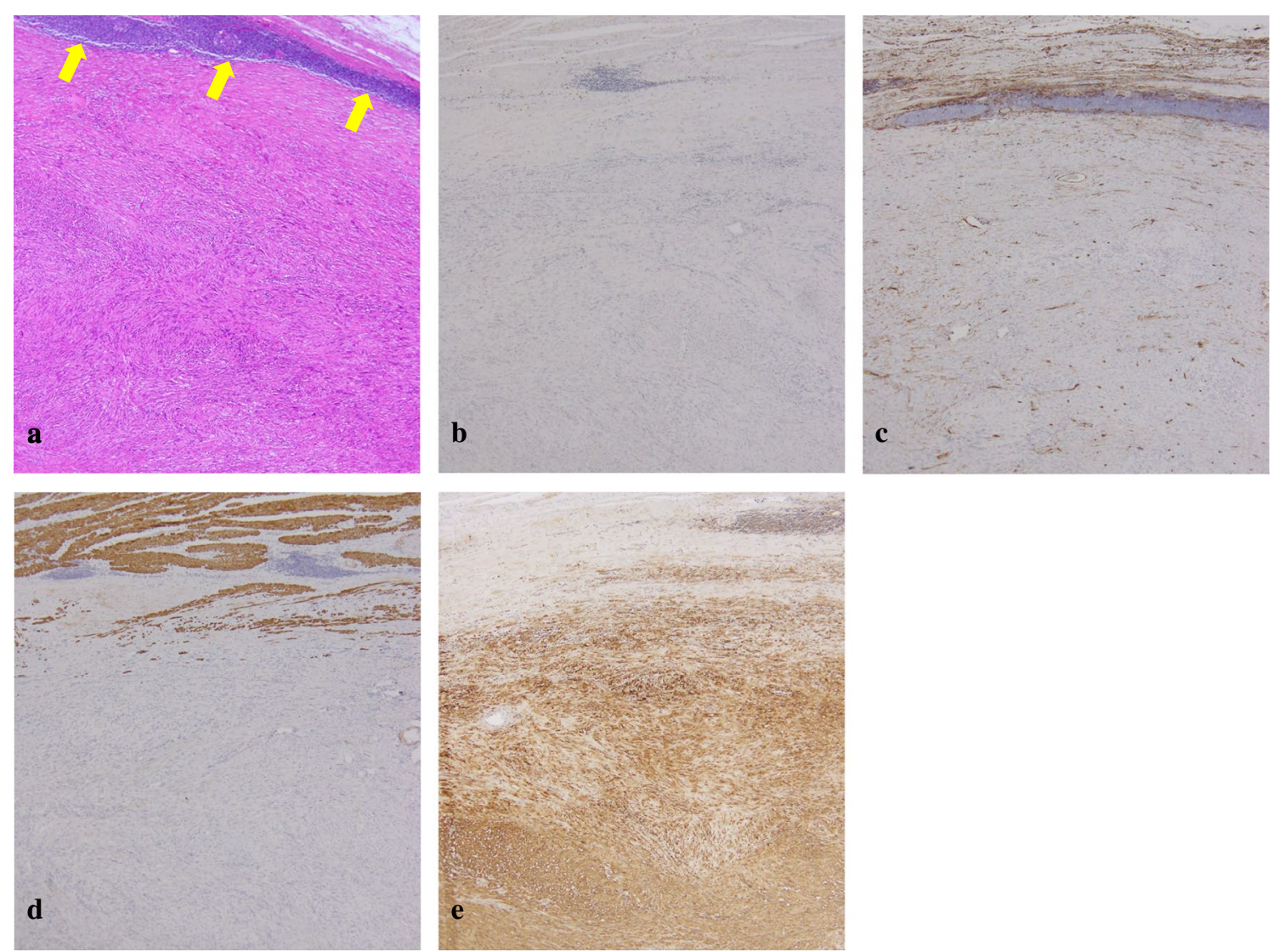

Fig. 3 a Hematoxylin-eosin staining demonstrating a bundle-like growth of the spindle-shaped tumor cells with acidophilic cytoplasm. A peritumoral lymphoid cuff is recognized (yellow arrows). $\mathbf{b}-\mathbf{e}$ Immunohistochemical staining revealing that the tumor is negative for KIT (b), CD34 (c), and desmin (d) and positive for S-100 protein (e) 
to $2 \%$. Hematoxylin-eosin staining of the lymph node showed nodular proliferation of atypical lymphoid cells composed predominantly of centrocytes with admixed scattered centroblasts (Fig. 4a). Immunohistochemical staining revealed that the lymph node was positive for bcl-2, CD20, and CD79a and negative for CD3 and CD30 (Fig. 4b-f). These histopathological and immunohistochemical findings were consistent with a gastric schwannoma and FL. The centroblasts were noted 2 to 3 per 50 HPFs; therefore, histological grading of FL was Grade $1[9]$.

The patient had an uneventful postoperative course and was discharged from our hospital on postoperative day 9. Postoperative positron emission tomography (PET) revealed no significant accumulation of ${ }^{18} \mathrm{~F}$-fluorodeoxyglucose. Bone marrow aspiration indicated no bone marrow invasion of FL. The patient was diagnosed with a grading of stage I according to the Ann Arbor classification. The Groupe d'Etude des Lymphomes Folliculaires (GELF) criteria were applicable as low-tumor burden FL and the patient was asymptomatic; therefore, the watchful waiting approach was decided upon as follow-up [9]. The patient is doing well without recurrence of either the gastric schwannoma or FL 28 months postsurgery.

\section{Discussion}

Schwannomas are neurogenic tumors originating from Schwann cells of a peripheral nerve sheath. They rarely occur in the gastrointestinal tract, with the stomach being the most common location. Gastric schwannomas are normally benign, and malignant transformation, with potential for lymph node metastasis, is extremely rare [4]. The majority of the swollen lymph nodes associated with gastric schwannomas are non-metastatic lymphadenopathy. In this paper, we report the case of a patient with a swollen lymph node of gastric schwannoma diagnosed with FL.

In the present study, the resected tumor histologically showed a peritumoral lymphoid cuff, which is known as a specific morphological finding of schwannoma, present in $78.8 \%-96.0 \%$ of gastric schwannomas [10-12].
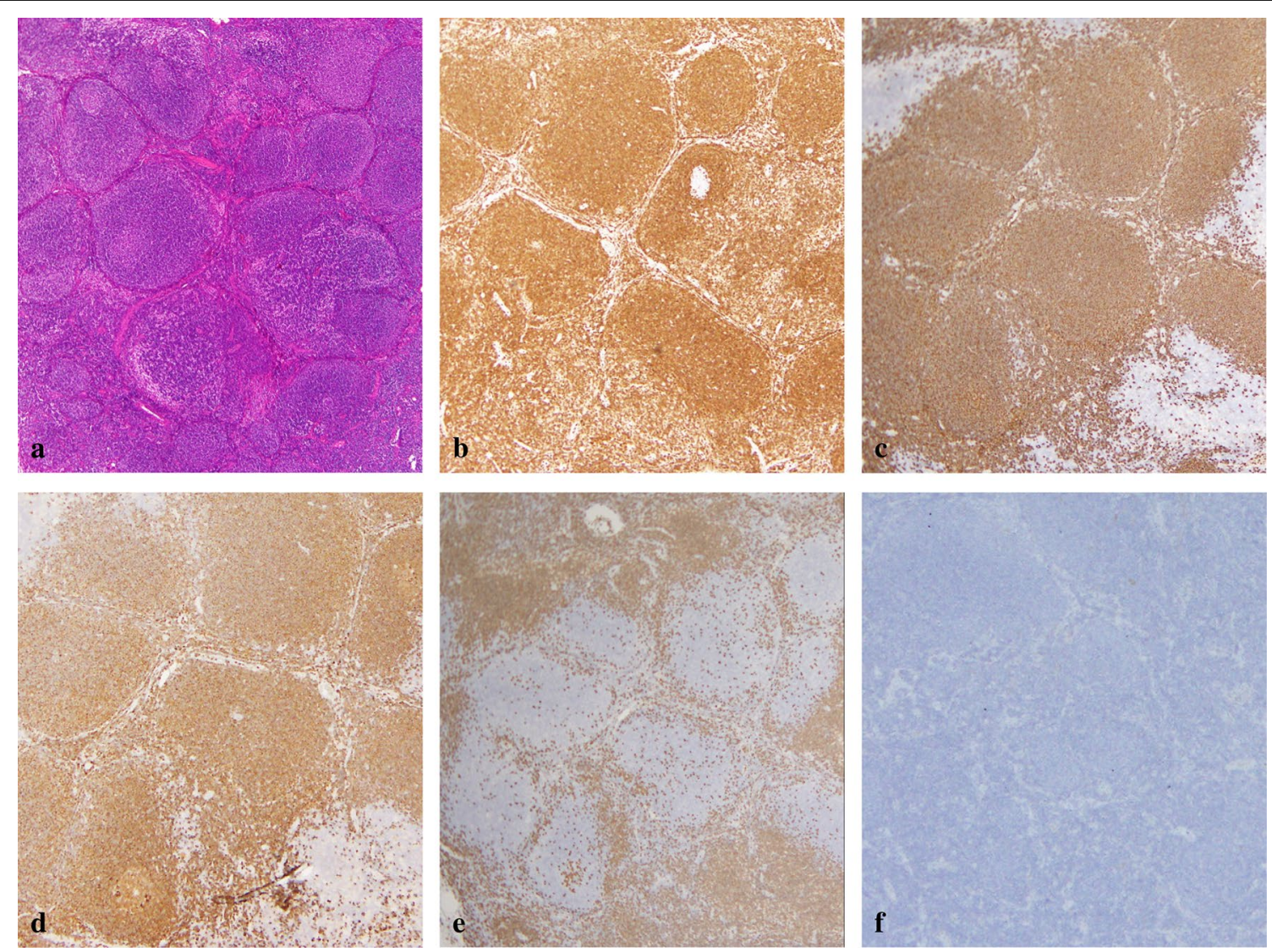

Fig. 4 a Hematoxylin-eosin staining of the lymph node showing nodular proliferation of atypical lymphoid cells composed predominantly of centrocytes with admixed scattered centroblasts. b-f Immunohistochemical staining revealing that the lymph node is positive for bcl-2 (b), CD20 (c), and CD79a (d) and negative for CD3 (e) and CD30 (f) 
Hou et al. speculate that the lymphoid cuff may be the result of cytokines secreted by the tumor cells that induce chemokinesis of lymphocytes [13]. This theory is supported by a recent paper; Bae et al. reported that a peritumoral lymphoid cuff of the main tumor positively correlates with the presence and size of regional lymphadenopathy in gastric schwannomas [12]. Regional lymphadenopathy is more commonly noted in gastric schwannomas than in other gastric SMTs [2, 3].

Malignant lymphoma, including FL, is usually noticed by the appearance of B symptoms and/or cervical, axillary, or inguinal swollen lymph nodes [14]. After the imaging test, malignant lymphoma is diagnosed by the excisional biopsy of a cervical, axillary, mesenteric, inguinal, mediastinal, or para-aortic swollen lymph node. Elevation of serum LDH and sIL-2R level, and abnormality of WBC count are helpful for diagnosis. Bulky lymphadenopathy growing around vessels, called the "sandwich sign," is known as a specific CT feature of the advanced malignant lymphoma [15]. In the present study, FL was coincidentally diagnosed, although there were no B symptoms, blood test abnormality, or significantly swollen lymph nodes on CT.

Primary lesion resection is usually performed for the gastric schwannoma; however, there is no consensus for the operative procedure of gastric schwannoma with swollen lymph nodes. In clinical practice, gastrectomy with lymph node dissection is occasionally performed when swollen lymph nodes are accompanied with gastric schwannomas, due to concern about the possibility of metastasis from the main tumor. However, most cases actually have no pathological lymph node metastasis [5-7]. This means that the routine lymph node dissection for the swollen lymph nodes of gastric schwannoma is unnecessary in most cases and can be an excessive invasion. Lymph node dissection is not necessary when the swollen lymph node is either non-metastatic or malignant lymphoma. From a clinical perspective, to avoid excessive invasion, the lymph node's metastatic potential should be determined prior to nodal dissection. If instant diagnosis of malignant lymphoma can become enabled in the future, we propose that the patients undergo intraoperative pathological diagnosis for swollen lymph nodes of gastric SMTs, including schwannoma. This may make it possible to avoid unnecessary lymph node dissection.

In the present case, the patient was asymptomatic and coincidently presented with Ann Arbor stage I and lowtumor burden FL. The patient received no initial therapy for FL. FL is the most common indolent B-cell nonHodgkin's lymphoma, most of which are diagnosed in an advanced stage (Ann Arbor stage III/IV) $[14,16]$. Limited stage (Ann Arbor I/II) FL is relatively uncommon; therefore, high-quality evidence for the treatment of limited stage FL is lacking [16]. Previous reports in the United States showed that the initial therapy given for stage I patients was as follows: rituximab plus chemotherapy, $28 \%$; radiation therapy, $27 \%$; observation, $17 \%$; systemic therapy plus radiation therapy, 13\%; rituximab monotherapy, 12\%; and other, 3\% [17]. Selected limited stage patients, such as asymptomatic and low-tumor burden patients, can be candidates for the strategy of a watchful waiting approach $[9,14]$. This is consistent with our present case. Advani et al. reported that stage I/II patients who received no initial therapy demonstrated that $63 \%$ of patients did not require treatment at a median follow-up of 86 months, and the estimated survival at 10 years was $85 \%$ [18]. Friedberg et al. reported that stage I patients managed with no initial therapy did not have a worse progression-free survival than those managed with radiation therapy [17].

The prognosis for an individual FL patient can be estimated by the Follicular Lymphoma International Prognostic Index (FLIPI), which is based on clinical and laboratory findings [9]. Five adverse prognostic factors of FLIPI were selected: age ( $>60$ years vs $\leq 60$ years), Ann Arbor stage (III/IV vs I/II), hemoglobin level $(<12 \mathrm{~g} / \mathrm{dL}$ $\mathrm{vs} \geq 12 \mathrm{~g} / \mathrm{dL}$ ), number of nodal areas $(>4 \mathrm{vs} \leq 4)$, and serum LDH level (above normal vs normal or below). Three risk groups were defined: low ( $0-1$ adverse factor), intermediate ( 2 adverse factors), and poor ( $\geq 3$ adverse factors). In our case, one adverse factor ( $>60$ years) was applicable and the risk group was low. On the other hand, gastric schwannomas are normally benign; even those over $100 \mathrm{~mm}$ in size and with a mitotic rate greater than 5/50 HPFs showed no evidence of aggressive behavior [10]. The benign gastric schwannoma rarely recurs after complete surgical resection [19]. In this case, the patient was diagnosed with a gastric schwannoma $80 \mathrm{~mm}$ in size with the mitotic count 0 to 1 per 50 HPFs, which was completely resected. Concerning the FL and gastric schwannoma in this case, long-term survival is expected to be good.

As this was a single-patient case report, these findings need to be confirmed by the accumulation of prospective evidence from more patients in multiple institutions. Gastric schwannomas are relatively rare; therefore, the number of patients treated in a single institution is limited. However, the current findings provide important information that can contribute to the development of a treatment strategy for gastric schwannomas accompanied with swollen lymph nodes.

\section{Conclusions}

The present report detailed an extremely rare case of FL coincidentally discovered in the swollen regional lymph node of gastric schwannoma. Gastric schwannomas are 
often accompanied by swollen regional lymph nodes, and many surgeons are confused regarding the need for lymph node dissection for such cases. This case indicates that when encountering a gastric schwannoma with regional lymphadenopathy, surgeons need to consider the possibility of malignant lymphoma and refrain from performing unnecessary lymph node dissection.

\section{Abbreviations}

CT: Computed tomography; FL: Follicular lymphoma; FLIPI: Follicular lymphoma international prognostic index; HPF: High-power field; LDH: Lactate dehydrogenase; PET: Positron emission tomography; IIL-2R: Soluble interleukin-2 receptor; SMT: Submucosal tumor; WBC: White blood cell.

\section{Acknowledgements}

The authors would like to thank Enago (www.enago.jp) for the English language review.

\section{Authors' contributions}

SF designed the study and drafted the manuscript. SF and TK performed the operation. TW and YO performed the histopathological examination. $\mathrm{HH}$ performed the hematological examination. TT, AG, $\mathrm{HT}, \mathrm{KO}, \mathrm{KK}, \mathrm{KH}, \mathrm{HI}, \mathrm{JH}, \mathrm{MY}$, and $\mathrm{Ml}$ participated in the manuscript revision process. All authors read and approved the final manuscript.

\section{Funding}

This research did not receive any specific grant from funding agencies in the public, commercial, or not-for-profit sectors.

\section{Availability of data and materials}

All data generated or analyzed during this study are included in this published article.

\section{Ethics approval and consent to participate}

This study was approved by the Ethics Committee of our institution (approval number: 20-6). Written informed consent was obtained from our patient.

\section{Consent for publication}

Written informed consent was obtained from our patient.

\section{Competing interests}

The authors declare that they have no competing interests.

\section{Author details}

1 Department of Gastroenterological Surgery, Kindai University Nara Hospital, 1248-1, Otoda-cho, Ikoma, Nara 630-0293, Japan. ${ }^{2}$ Department of Pathology, Kindai University Nara Hospital, Nara, Japan. ${ }^{3}$ Department of Hematology, Kindai University Nara Hospital, Nara, Japan.

Received: 2 June 2020 Accepted: 18 September 2020 Published online: 29 September 2020

\section{References}

1. Fujiwara S, Nakajima K, Nishida T, Takahashi T, Kurokawa Y, Yamasaki M, et al. Gastric schwannomas revisited: has precise preoperative diagnosis become feasible? Gastric Cancer. 2013;16:318-23.
2. Min YW, Park HN, Min BH, Choi D, Kim KM, Kim S. Preoperative predictive factors for gastrointestinal stromal tumors: analysis of 375 surgically resected gastric subepithelial tumors. J Gastrointest Surg. 2015;19:631-8.

3. Choi YR, Kim SH, Kim SA, Shin Cl, Kim HJ, Kim SH, et al. Differentiation of large $(\geq 5 \mathrm{~cm})$ gastrointestinal stromal tumors from benign subepithelial tumors in the stomach: radiologists' performance using CT. Eur J Radiol. 2014;83:250-60.

4. Hu BG, Wu FJ, Zhu J, Li XM, Li YM, Feng Y, et al. Gastric schwannoma: a tumor must be included in differential diagnoses of gastric submucosal tumors. Case Rep Gastrointest Med. 2017;2017:9615359.

5. Namikawa T, Kobayashi M, Hanazaki K. Gastric schwannoma with regional lymphadenopathy. Clin Gastroenterol Hepatol. 2017;15:e145-e146.

6. Shimizu S, Saito H, Kono Y, Murakami Y, Kuroda H, Matsunaga T, et al. Gastric schwannoma with enlargement of the regional lymph nodes resected using laparoscopic distal gastrectomy: report of a patient. Yonago Acta Med. 2017;60:59-63.

7. Tang C, Pan Q, Xu Z, Zhou X, Wang Y. Gastric schwannoma with giant ulcer and lymphadenopathy mimicking gastric cancer: a case report. BMC Gastroenterol. 2020;20:36.

8. Nishida T, Kawai N, Yamaguchi S, Nishida Y. Submucosal tumors: comprehensive guide for the diagnosis and therapy of gastrointestinal submucosal tumors. Dig Endosc. 2013;25:479-89.

9. Kahl BS, Yang DT. Follicular lymphoma: evolving therapeutic strategies. Blood. 2016;127:2055-63.

10. Voltaggio L, Murray R, Lasota J, Miettinen M. Gastric schwannoma: a clinicopathologic study of 51 cases and critical review of the literature. Hum Pathol. 2012;43:650-9.

11. Tao K, Chang W, Zhao E, Deng R, Gao J, Cai K, et al. Clinicopathologic features of gastric schwannoma: 8-year experience at a single institution in China. Medicine (Baltimore). 2015;94:e1970.

12. Bae H, Van Vrancken M, Kang TW, Park HY, Chu J, Park HK, et al. Peritumoral lymphoid cuff correlates well with lymph node enlargement in gastrointestinal schwannomas. Oncotarget. 2018;9:12591-8.

13. Hou YY, Tan YS, Xu JF, Wang XN, Lu SH, Ji Y, et al. Schwannoma of the gastrointestinal tract: a clinicopathological, immunohistochemical and ultrastructural study of 33 cases. Histopathology. 2006;48:536-45.

14. Freedman A, Jacobsen E. Follicular lymphoma: 2020 update on diagnosis and management. Am J Hematol. 2020;95:316-27.

15. Hardy SM. The sandwich sign. Radiology. 2003;226:651-2.

16. Sorigue M, Tuset V, Sancho JM. Treatment of localized-stage follicular lymphoma. Eur J Haematol. 2018;101:245-56.

17. Friedberg JW, Byrtek M, Link BK, Flowers C, Taylor M, Hainsworth J, et al. Effectiveness of first-line management strategies for stage I follicular lymphoma: analysis of the National LymphoCare Study. J Clin Oncol. 2012;30:3368-75

18. Advani R, Rosenberg SA, Horning SJ. Stage I and II follicular non-Hodgkin's lymphoma: long-term follow-up of no initial therapy. J Clin Oncol. 2004;22:1454-9.

19. Hong $X$, Wu W, Wang M, Liao Q, Zhao Y. Benign gastric schwannoma: how long should we follow up to monitor the recurrence? A case report and comprehensive review of literature of 137 cases. Int Surg. 2015;100:744-7.

\section{Publisher's Note}

Springer Nature remains neutral with regard to jurisdictional claims in published maps and institutional affiliations. 